

Cite this: Phys. Chem. Chem. Phys., 2016, 18, 18024

Received 20th April 2016 Accepted 13th June 2016 DOI: $10.1039 / c 6 c p 02634 f$

www.rsc.org/pccp

\section{Metal ion-humic acid nanoparticle interactions: role of both complexation and condensation mechanisms $\dagger$}

\begin{abstract}
Raewyn M. Town ${ }^{\star a}$ and Herman P. van Leeuwen ${ }^{b}$
Purely Donnan type models for electrostatic binding by humic acid (HA) nanoparticles are shown to be physically incomplete. To describe the extent of ion binding by HA, such models need to invoke parameters that are not consistent with experimental observations. These disparate parameters include anomalously high Donnan potentials, as well as intrinsic affinity constants for electrostatically associating ions such as $\mathrm{Ca}^{2+}$. In contrast, the recently introduced counterion condensation - Donnan model (CCD) provides a physicochemically realistic description of the electrostatic contribution to metal ion binding by humic acid nanoparticles. The extent of $\mathrm{Ca}^{2+}-\mathrm{HA}$ association can be adequately described solely in terms of electrostatics only, including counterion condensation in the intraparticulate double layer in addition to Donnan partitioning in the remainder of the particle body. The binding of $\mathrm{Cd}(॥), \mathrm{Pb},(॥)$ and $\mathrm{Cu}(॥)$ by $\mathrm{HA}$ also involves inner-sphere complex formation leading to intraparticulate metal species distributions with major proportions of condensed and complexed ions.
\end{abstract}

\section{Introduction}

Humic acids (HA) are ubiquitous in soils and natural waters and form an important complexant for a wide range of ions and small molecules. ${ }^{1}$ Accordingly, there is a wealth of literature on the measurement and modelling of its physicochemical association properties. $^{1-3}$ Aqueous HA can be considered to be composed of soft nanoparticles (NP) that carry a net negative charge at ambient $\mathrm{pH}^{4,5}$ Indeed, the large number of ionisable functional groups generate a significant particle electric field, which in itself can influence the dynamics and extent of its interactions with oppositely charged ions, $\mathrm{M}^{z+} \cdot{ }^{6}$ Rigorous interpretation of physicochemical M-HA interactions must therefore take proper account of electrostatic interactions as well as intrinsic chemical contributions to the binding.

Conventionally, M-HA complex formation is described in terms of apparent stability constants, $\bar{K}_{\text {app }}$, that are defined in terms of concentrations of chemical species that are averaged over the entire volume of the solution/dispersion. Furthermore, so-called 'bound' $\mathbf{M}$ is collectively taken as all forms of $\mathbf{M}$ that are associated with the HA entity, including intraparticulate

\footnotetext{
${ }^{a}$ Department of Physics, Chemistry and Pharmacy, University of Southern Denmark, Campusvej 55, 5230 Odense, Denmark.E-mail: raewyn.town@sdu.dk

${ }^{b}$ Physical Chemistry and Soft Matter, Wageningen University \& Research, Stippeneng 4, 6708 WE Wageningen, The Netherlands

$\dagger$ Electronic supplementary information (ESI) available. See DOI: 10.1039/ с6ср02634f
}

free $\mathbf{M}$ and even the excess free $\mathbf{M}$ in the extraparticulate interfacial double layer. At this level, various equilibrium models for metal ion speciation have been developed, e.g. the NICA-Donnan ${ }^{2}$ and WHAM $^{3}$ models. Generally the electrostatic contribution to $\mathrm{M}^{z^{+}}$binding by $\mathrm{HA}$ is modeled by assuming Donnan-type partitioning into the HA body or Boltzmann-type accumulation in the extraparticulate zone. ${ }^{3,7-12}$ The relevant physicochemical parameters then are the effective Donnan volume and the charge density of the HA particle. The various affinities of the functional $\mathbf{M}^{z+}$ binding sites are modeled either by a number of different types of discrete sites, e.g. WHAM, ${ }^{3}$ or by a continuous distribution of site affinities, e.g. NICA-Donnan. ${ }^{2}$ Evidently the meaning of $\bar{K}_{\text {app }}$ values is not transparent for the case of nanoparticulate complexants in which binding sites are confined to the particle body within which the local conditions are significantly different from those in the bulk aqueous medium. For example, $\bar{K}_{\text {app }}$ values increase as the ionic strength of the bulk aqueous medium decreases. ${ }^{13,14}$ This observation is not due to a significant change in the intrinsic chemical affinity, but rather is a consequence of the greater free $\mathrm{M}$ concentration within the HA entity at lower ionic strength. Obviously, a more rigorous description of $\mathrm{M}$-HA complex formation requires detailed knowledge of both electrostatic and intrinsic chemical affinity contributions to the binding and the ensuing consequences for the detailed metal ion speciation inside the HA particle entity.

Recently the nature of electrostatic binding by HA has been reconsidered. ${ }^{15}$ The typically high structural charge densities, 
that prevail within the particle body at ambient $\mathrm{pH}$, invoke cooperative electrostatics effects such as counterion condensation, which features a large preference for the higher valency cations. Such phenomena have been described in detail by Manning for linear polyelectrolytes, ${ }^{16,17}$ and refer to the tendency of the polyion to condense counterions in its immediate vicinity if the structural charge density is beyond certain limiting values. Counterion condensation is applicable to polyions of any geometry with sufficient charge density, e.g. it has been reported for polyelectrolytes such as DNA, ${ }^{16}$ soft nanoparticles such as dendrimers, ${ }^{18-20}$ as well as core-shell NPs with a 3D structural charge in the shell. ${ }^{21}$ We have observed that counterion condensation in the 3D site distribution structure occurs at charge separations greater than for the $1 \mathrm{D}$ case. ${ }^{15}$ For HA, conductivity data have shown that divalent ions such as $\mathrm{Ca}^{2+}$ and $\mathrm{Ba}^{2+}$ exhibit condensation behavior, whilst monovalent ions do not. ${ }^{22}$ Notably, it was shown that the extent of electrostatic association of $\mathrm{Ca}^{2+}$ with $\mathrm{HA}$ cannot be explained by Donnan-partitioning alone. The CCD model therefore combines counterion condensation in the intraparticulate double layer with Donnan partitioning in the remainder of the particle body. It well describes the extent to which $\mathrm{Ca}^{2+}$ associates with a range of HA samples with different particle sizes at various ionic strengths. ${ }^{15}$ It has also been applied to the intraparticulate speciation analysis of complexes that involve covalency, i.e. those of $\mathrm{Cd}(\mathrm{II}), \mathrm{Pb}(\mathrm{II})$, and $\mathrm{Cu}(\mathrm{II}) .^{23}$ Three types of intraparticulate metal species then are distinguished, namely free hydrated ions, electrostatically condensed ions, and inner-sphere complexes. Here we compare the CCD description of M-HA interactions with the results generated by equilibrium speciation models that consider Donnan-type partitioning to be the only electrostatic contribution to the binding. We explore the nature of the fundamental parameters that are relevant for defining the extent of complexation, e.g. the effective Donnan volume and the applicable charge density, and assess the outcomes in terms of the physical consistency.

\section{Theory}

The equilibrium relationship between the concentrations of the metal ion and the various binding sites of the chemically heterogeneous HA complexant is described by a distributed affinity. The apparent stability constants $\bar{K}_{\text {app }}$ for the complexes are expressed in terms of smeared-out concentrations and include both electrostatic and intrinsic chemical contributions to the binding between a metal ion $\mathrm{M}$ and a reactive site, $\mathrm{S}$. The magnitude of $\bar{K}_{\text {app }}$ depends on the degree of occupation, $\theta_{\mathbf{M}}$, of the binding sites by $\mathbf{M}^{1}{ }^{1}$ We define $\theta_{\mathbf{M}}$ as the ratio between the concentrations of inner-sphere metal complexes, MS, and reactive sites, i.e.

$$
\theta_{\mathrm{M}}=c_{\mathrm{MS}} / c_{\mathrm{S}, \mathrm{t}}=c_{\mathrm{MS}}^{*} / c_{\mathrm{S}, \mathrm{t}}^{*}
$$

where $c_{\mathrm{MS}}$ and $c_{\mathrm{S}, \mathrm{t}}$ are the local intraparticulate concentrations of the inner-sphere complexes and total reactive sites, respectively, and $c_{\mathrm{MS}}^{*}$ and $c_{\mathrm{S}, \mathrm{t}}^{*}$ are the smeared-out counterparts.

\subsection{Intrinsic stability constants}

The intrinsic stability constant $K_{\text {int }}$ represents the inherent chemical affinity between $\mathrm{M}$ and $\mathrm{S}$, not including the long-range electrostatics beyond those on the scale of atom-atom interactions. $K_{\text {int }}$ values may only be obtained from $K_{\text {app }}$ after applying the appropriate correction for the electrostatic contribution to the binding. For simple ligands, the outer-sphere stability constant, $K^{\mathrm{os}}$, represents the magnitude of the electrostatic attraction between a negatively charged reactive site and a positively charged metal ion. The extent to which the concentration of the reactant metal ions in the outer-sphere volume is enhanced relative to that in the bulk electrolyte medium, $f^{\text {os }}$, can be computed on the basis of Fuoss-Boltzmann electrostatics. ${ }^{24,25}$

$$
f^{\mathrm{os}}=\exp \left(-U^{\mathrm{os}} / k T\right)
$$

where $U^{\text {os }}$ is the interionic potential for an individual ion pair, given by:

$$
U^{\mathrm{os}}=\frac{z_{\mathrm{M}} z_{\mathrm{S}} e^{2}}{4 \pi \varepsilon_{0} \varepsilon a}\left(1-\frac{\kappa a}{1+\kappa a}\right)
$$

where $a$ is the center-to-center distance between $\mathrm{M}$ and $\mathrm{S}, z_{\mathrm{M}}$ and $z_{\mathrm{S}}$ are the charge on the metal ion and the binding site, respectively, and $\varepsilon_{0} \varepsilon$ is the permittivity of the electrolyte solution. It follows that for simple ligands:

$$
K_{\mathrm{int}}=K / f^{\mathrm{os}}
$$

where $K$ is the conventional stability constant for a simple ligand. E.g. for a $2+/ 1-$ ion pair with a center-to-center distance of $0.6 \mathrm{~nm}$, the values of $f^{\text {os }}$ are approximately 4 and 7 for ionic strengths of 100 and $10 \mathrm{~mol} \mathrm{~m}^{-3}$, respectively.

For the case of soft charged nanoparticulate complexants, the magnitude of the particle's electric field determines the extent to which oppositely charged ions electrostatically associate with the NP entity. The majority of literature for humic acids assumes that this association takes the form of Donnan partitioning into the NP body. The establishment of a Donnan phase requires that the particle radius is much greater than the intraparticulate screening length, $\kappa_{\mathrm{p}}^{-1}, 33$ and that the average structural charge separation, $\ell_{\mathrm{C}}$, is sufficiently small so that $\kappa_{\mathrm{p}} \ell_{\mathrm{C}} \ll 1$, i.e. high charge density regime. ${ }^{5}$ When these conditions are met, a Donnan potential difference, $\psi_{\mathrm{D}}$, is established between the bulk of the soft phase and the bulk aqueous medium: ${ }^{26}$

$$
\psi_{\mathrm{D}}=\frac{R T}{z F} \operatorname{asinh}\left(\frac{\rho_{\mathrm{p}}}{2 z F c_{1}}\right)
$$

where $z_{-}=z_{+}=z$ is the valence of the symmetrical excess background electrolyte with bulk concentration $c_{1}, \rho_{\mathrm{p}}$ is the structural volume charge density due to charged groups on the backbone of the soft body, and other constants have their usual meaning. Expressions are also available for asymmetrical electrolytes. ${ }^{26}$ Any type of ion $i$, with valency $z_{i}$, will partition between the soft particle phase (where its concentration is $c_{\mathrm{i}, \mathrm{D}}$ ) and the electrolyte solution (where its concentration is $c_{\mathrm{i}}^{*}$ ) according to a Boltzmann factor, $\bar{f}_{\mathrm{B}}:{ }^{27}$

$$
\bar{f}_{\mathrm{B}, \mathrm{i}}=\frac{c_{\mathrm{i}, \mathrm{D}}}{c_{\mathrm{i}}^{*}}=\exp \left(\frac{-z_{\mathrm{i}} F \psi_{\mathrm{D}}}{R T}\right)
$$


When Donnan partitioning is the sole electrostatic contribution to the binding of $\mathrm{M}$ by $\mathrm{S}$, the $\bar{K}_{\text {int }}$ is simply given by:

$$
\bar{K}_{\text {int }}=\bar{K}_{\text {app }} / \bar{f}_{\mathrm{B}}
$$

However, recent work has provided evidence for additional electrostatic binding due to counterion condensation. A two-state approximative model was developed which combines Donnan partitioning within the uncharged bulk zone of the soft NP (volume $V_{\mathrm{D}}$ and volume fraction $\varphi_{\mathrm{D}}$ ) with counterion condensation in the strongly negatively charged intraparticulate double layer zone of thickness $\ell_{\mathrm{DL}}$ (with volume $V_{\mathrm{DL}}$ and volume fraction $\left.\varphi_{\mathrm{DL}}\right){ }^{15,23}$ The justification of a constant Donnan potential throughout the bulk core volume of the particle requires a volume fraction ratio $\varphi_{\mathrm{DL}} / \varphi_{\mathrm{D}}$ much less than unity, i.e. $\kappa_{\mathrm{p}} r_{\mathrm{p}}$ should be well above unity. In this work we focus on HA entities for which $\varphi_{\mathrm{DL}} / \varphi_{\mathrm{D}}$ is less than 0.1 . In the presence of counterion condensation, the distinction between $\bar{K}_{\text {app }}$ and $\bar{K}_{\text {int }}$ encompasses more than the Boltzmann correction of the free metal ion ( $c f$. eqn (7)). Rather, the intrinsic stability constant is defined in terms of intraparticulate (in situ) conditions and reactant concentrations, i.e.

$$
\bar{K}_{\text {int }}=\frac{c_{\mathrm{MS}}}{c_{\mathrm{S}} c_{\mathrm{M}, \mathrm{f}}}
$$

where $c_{\mathrm{MS}}, c_{\mathrm{M}, \mathrm{f}}$ and $c_{\mathrm{S}}$ are the local average concentrations of inner-sphere complexes, free metal ion and reactive sites in the particle body, respectively, and the bar in $\bar{K}_{\text {int }}$ signifies that, for the present case of a heterogeneous complexant, the intrinsic stability represents a weighted average of all the inner-sphere complexes that are formed at the applicable $\theta_{\mathbf{M}}$. The ensuing metal ion speciation scheme for high charge density soft NP complexants has been published previously, ${ }^{23}$ and is given in the ESI $\dagger$ (Table S1). We highlight that our approach makes no assumptions about the nature of the charged or reactive sites, nor about the metal binding isotherm.

\section{Experimental}

The experimental details for the data presented herein were reported previously. ${ }^{23}$ NICA-Donnan computations were performed using Visual Minteq 3.1 and the generic HA parameters. ${ }^{28}$ The local concentrations were determined from the smeared-out concentrations that were fitted by the model in each computation, together with the generic Donnan volume. For each type of complexing ion, NICA-Donnan assumes a bimodal affinity distribution: for the present comparative purposes, the sum of the complexes with both types of sites was used to compute the proportion of inner-sphere complexes.

\section{Results and discussion}

\subsection{Electrostatic contribution to the binding}

The physical size of the HA nanoparticle in aqueous dispersion is important for both the Donnan-only and the two-state CCD electrostatic models. A water content of $\mathrm{ca}$. $80 \%$ has been reported for several types of $\mathrm{HA}$ from viscometric data at $\mathrm{pH} 7$ and ionic strengths of 10 and $100 \mathrm{~mol} \mathrm{~m}^{-3} \cdot{ }^{29}$ For establishment of the CCD approach, the water content of about $80 \%$ provided a consistent description of the $\mathrm{Ca}^{2+}$ association with several types of HA, including Aldrich HA, at ionic strengths of 10 and $100 \mathrm{~mol} \mathrm{~m}^{-3}$ [see ref. 15 and references therein]. This consistent water content at both ionic strengths is in line with experimental estimates of the particle size of various types of HA by dynamic light scattering, capillary electrophoresis, and fluorescence correlation spectroscopy which confirm that the HA particle size is practically independent of ionic strength in the range 0 to $200 \mathrm{mM}^{30-32}$ Due to the presence of a large density of structural charged sites with insignificant local mobility, there is uncertainty about the magnitude of the effective intraparticulate Debye length $\kappa_{\mathrm{p}}^{-1}$. Duval ${ }^{33}$ has made some estimations of this crucial parameter which come to approximately $0.6 \mathrm{~nm}$ for both 10 and $100 \mathrm{~mol} \mathrm{~m}^{-3}$ ionic strength in the surrounding medium. The binding of $\mathrm{Ca}^{2+}$ by the HA studied here has been interpreted in terms of a thickness of the electric condensation zone, $\ell_{\mathrm{DL}}$, for which the magnitude was obtained from fitting to agreement with the potential change over the intraparticulate part of the interfacial double layer. ${ }^{15}$ There should be a close relationship between $\ell_{\mathrm{DL}}$ and $\kappa_{\mathrm{p}}$ but to our knowledge this has not yet been formulated. For the time being we can proceed by accepting the magnitude of $\ell_{\mathrm{DL}}$ together with the degree of $\mathrm{Ca}^{2+}$ condensation as the leading numbers for the condensation of any other divalent metal ion. See Section 4.2 below for details. Accordingly, within the total particle volume, $V_{\mathrm{p}}$, the CCD model distinguishes between an intraparticulate double layer shell with volume $V_{\mathrm{DL}}$, and a Donnan phase with volume $V_{\mathrm{D}}=V_{\mathrm{p}}-V_{\mathrm{DL}}$. For the present system with $\kappa_{\mathrm{p}} r_{\mathrm{p}} \gg 1$ and both ionic strengths investigated, $V_{\mathrm{D}}$ is close to the total particle volume.

The Donnan volume used in the NICA-Donnan model is obtained by fitting a combination of $V_{\mathrm{D}}$ and $\psi_{\mathrm{D}}$ to a so-called mastercurve for protolytic titrations of $\mathrm{HA}^{7}$ An empirical double logarithmic expression links the Donnan volume to the ionic strength of the bulk medium: ${ }^{12}$

$$
\log V_{\mathrm{D}}=b(1-\log I)-1
$$

where $b$ is a fitting parameter and $I$ the ionic strength of the medium. Thus eqn (9) indicates that $V_{\mathrm{D}}$ is significantly dependent on $I$, even though $V_{\mathrm{p}}$ is practically independent of $I$ (see above). Apart from this discrepancy, the physical reasonableness of the NICA-Donnan $V_{\mathrm{D}}$ and $\psi_{\mathrm{D}}$ values has been questioned on the basis that the generic values for $\psi_{\mathrm{D}}$ are significantly more negative than those experimentally determined for HA, and the concomitantly fitted protonation constants are anomalously low. ${ }^{34,35}$ It should also be noted that the approach used to obtain the NICA-Donnan $V_{\mathrm{D}}, \psi_{\mathrm{D}}$ couple involves covariance of several parameters and the outcome is sensitive to how the fitting is implemented. For example, a modified procedure that minimizes the covariance of the NICADonnan parameters and optimizes the $b$ value for each ionic strength, yields protonation constants that are in better agreement with literature data, in combination with a different $b .^{34}$

In passing we note that the WHAM speciation model assumes Donnan partitioning to occur only within the extraparticulate double layer, irrespective of the particle size. ${ }^{3}$ The occurrence of 
extraparticulate "Donnan partitioning" is also allowed in the NICA-Donnan model for fulvic acids and small humic acids for which the fitted Donnan volume is allowed to be greater than the particle size. ${ }^{36}$ However, the extraparticulate double layer carries a net charge density and does not contain any fixed charges at all, i.e. it is not a true Donnan phase. Rather, Boltzmann accumulation should be expected to occur in such zones. For $\kappa_{\mathrm{p}} r_{\mathrm{p}}$ of order unity and lower, the amount of $\mathrm{M}^{2+}$ in the extraparticulate DL may indeed be significant in the total amount of $\mathrm{M}$ associated with the particle. In the CCD, the excess amount of $\mathbf{M}$ in the extraparticulate DL is straightforwardly obtained from the Boltzmann accumulation of $\mathrm{M}^{2+}$ as ensuing from the diffuse double layer potential profile ${ }^{37}$ and included in the mass balance for particle-associated free $\mathbf{M}$.

The approaches for modeling the electrostatic contribution to cation binding by HA are summarized in Table 1 below.

The equilibrium speciation in a range of M-HA systems has been modeled by the NICA-Donnan approach, with typically good fits to the experimentally measured free metal ion concentrations being reported. ${ }^{38}$ Despite their very different constituent parameters, the WHAM and NICA-Donnan models predict similar concentrations of free metal ions in the presence of dissolved organic matter. ${ }^{39}$ This observation points to the empirical nature of the models. The quality-of-fit of the models is assessed on the basis of agreement between the measured and computed 'free' versus 'bound' $\mathrm{M}$, where 'bound' includes all forms of $\mathrm{M}$ associated with the HA entities. However, the physical meaning of the various parameters in these equilibrium models is obscured due to the concomitant nature of the fitting process, and the interrelationships between the sub-models for intrinsic chemical and electrostatic binding components. ${ }^{28}$ Indeed, a recent review of the NICA-Donnan approach stated "The fact that a set of parameter values can be non-unique is first of all due to the large number of parameters in the model. A different way of fitting of the parameters to the data may lead to an equally good description of the data, but different parameter values.",

\subsection{Speciation in the electrostatic $\mathrm{Ca}^{2+}-\mathrm{HA}$ system}

Here we seek to identify the physicochemically most reasonable descriptors for the binding of $\mathrm{M}^{2+}$ by HA. As a straightforward starting point, we consider the case of $\mathrm{Ca}^{2+}$ association with HA. The associative reactions of $\mathrm{Ca}^{2+}$ with negatively charged functional groups are dominated by electrostatics. ${ }^{40-42}$ The extent to which $\mathrm{Ca}^{2+}$ associates with $\mathrm{HA}$ was found to be far greater than that predicted by only Donnan-type electrostatics, with more highly charged HAs exhibiting the greatest amount of $\mathrm{Ca}^{2+}$ association. ${ }^{15}$ Accordingly, in order to describe the $\mathrm{Ca}^{2+}$-HA data, the NICA-Donnan model resorts to inclusion of intrinsic affinity constants for $\mathrm{Ca}^{2+}$ complexation, together with $V_{\mathrm{D}}$ values that are smaller for more highly charged humic acids. For example, the NICA-Donnan $V_{\mathrm{D}}$ values fitted to $\mathrm{Ca}^{2+}-\mathrm{HA}$ binding data follow the inverse order of the HA charge density: e.g. for Elliot soil HA with carboxyl content $4.8 \mathrm{~mol} \mathrm{~kg}^{-1}, V_{\mathrm{D}}$ is $0.23 \times 10^{-3} \mathrm{~m}^{3} \mathrm{~kg}^{-1}$, with a concomitant protonation constant $\left(K_{1, \mathrm{H}}\right)$ of $10^{-0.8} \mathrm{~m}^{3} \mathrm{~mol}^{-1}$ significantly lower than the generic value of $10^{-0.1} \mathrm{~mol} \mathrm{~m}^{-3}$, 3 whilst for a forest soil HA with carboxyl content $2.18 \mathrm{~mol} \mathrm{~kg}^{-1}, V_{\mathrm{D}}$ is $2.95 \times 10^{-3} \mathrm{~m}^{3} \mathrm{~kg}^{-1}$, using the generic protonation constants. ${ }^{44}$ An example of the speciation computed by the NICA-Donnan model for the $\mathrm{Ca}^{2+}$ HA system is given in Table 2 . The fit to the data invokes a $\psi_{\mathrm{D}}$ of ca. $-100 \mathrm{mV}$, which is rather negative for the given ionic strength of $82 \mathrm{~mol} \mathrm{~m}^{-3,45}$ as well as inner-sphere $\mathrm{Ca}^{2+}$ complex formation. As much as $81 \%$ of the particle-associated $\mathrm{Ca}^{2+}$ is predicted to be inner-sphere bound, whereas the $K_{\text {int }}$ for CaHA is similar to its purely electrostatic $K^{\mathrm{os}}$ which would hardly suggest any inner-sphere complexes at all.

In contrast, the CCD model describes the extent of $\mathrm{Ca}^{2+}-\mathrm{HA}$ association for a range of HA samples, $\mathrm{pH}$ and ionic strength values, via inclusion of the mere electrostatic contributions due to counterion condensation within the intraparticulate double layer, together with a more realistic $\psi_{\mathrm{D}}$ of $-70 \mathrm{mV}$ in the bulk of the particle body (Table 2).

\subsection{Intraparticulate speciation with inner-sphere complexes M-HA}

Here we consider the speciation in M-HA systems in which intrinsic chemical binding is also involved, and compare the results obtained by the CCD and NICA-Donnan approaches. The parameters defining the CCD speciation scheme are given in the ESI. $\uparrow$ Table 3 collates the concentrations of the various metal species for the case of a 1-1 background electrolyte $\left(\mathrm{KNO}_{3}\right)$. In the CCD case, the given intraparticulate $\bar{K}_{\text {int }}$ values are those derived from local concentrations after accounting for the electrostatic effects (eqn (8)), whilst the NICA-Donnan values are obtained via eqn (7).

The data show that for both approaches the free hydrated metal ions generally are a minor proportion of the total intraparticulate M. The CCD results for Cd(II) at $I=100 \mathrm{~mol} \mathrm{~m}^{-3}$, show that $70 \%$ of the uncomplexed $\mathrm{M}$ is condensed in the intraparticulate double layer and $30 \%$ is inner-sphere bound,

Table 1 Models for electrostatic binding by humic acid

\begin{tabular}{|c|c|c|}
\hline Model & Electrostatic binding & Donnan volume, $V_{\mathrm{D}}$, relative to particle volume, $V_{\mathrm{p}}$ \\
\hline WHAM $^{3}$ & Donnan partitioning ${ }^{a}$ & $V_{\mathrm{D}}$ entirely extraparticulate $V_{\mathrm{D}}=\frac{4 \pi N_{\mathrm{Av}}}{3}\left[\left(r_{\mathrm{p}}+\kappa^{-1}\right)^{3}-r_{\mathrm{p}}^{3}\right]$ \\
\hline NICA-Donnan ${ }^{2}$ & Donnan partitioning ${ }^{a}$ & $\begin{array}{l}V_{\mathrm{D}} \text { may be smaller or larger than the physical } V_{\mathrm{p}} \cdot \log V_{\mathrm{D}}=b(1-\log I)-1 \text {, } \\
\text { where } b \text { is an empirical factor with generic value of } 0.49 \text { for HA }\end{array}$ \\
\hline $\mathrm{CCD}^{23}$ & $\begin{array}{l}\text { Counterion condensation plus } \\
\text { Donnan partitioning }\end{array}$ & $V_{\mathrm{D}}=V_{\mathrm{P}}-V_{\mathrm{DL}}$ \\
\hline
\end{tabular}


Table 2 Speciation computed in the $\mathrm{Ca}^{2+}-\mathrm{HA}$ system by CCD and NICADonnan approaches. $c_{\mathrm{Ca}, \mathrm{t}}^{*}=0.195 \mathrm{~mol} \mathrm{~m}^{-3}, c_{\mathrm{HA}, \mathrm{t}}^{*}=2000 \mathrm{~g} \mathrm{~m}^{-3}, \mathrm{pH}=8$ and $I=82 \mathrm{~mol} \mathrm{~m}^{-3}$ in bulk electrolyte medium ${ }^{a}$

\begin{tabular}{lll}
\hline & $\mathrm{CCD}^{b}$ & NICA-Donnan \\
\hline$c_{\mathrm{Ca}, \mathrm{f}}^{*}, \mathrm{~mol} \mathrm{~m}^{-3}$ & $3.8 \times 10^{-3}$ & $7.8 \times 10^{-3}$ \\
$c_{\mathrm{Ca}, \mathrm{t}}, \mathrm{mol} \mathrm{m}{ }^{-3}$ & 38 & 88.5 \\
Fraction free in $V_{\mathrm{DL}}$ & $4 \times 10^{-3}$ & - \\
Fraction free in $V_{\mathrm{D}}$ & 0.03 & 0.19 \\
Fraction condensed in $V_{\mathrm{DL}}$ & 0.97 & - \\
Fraction inner-sphere MS & - & 0.81 \\
$\bar{f}_{\mathrm{B}, \mathrm{M}}$ in $V_{\mathrm{D}}$ & 250 & 2130
\end{tabular}

${ }^{a}$ Experimental data from Hering and Morel. ${ }^{46}{ }^{b}$ Computed using the experimentally measured concentration of free $\mathrm{Ca}^{2+}$ in the bulk medium, together with a condensation factor, $\bar{f}_{\mathrm{C}}$, of 0.8 in the intraparticulate double layer with thickness of $2 \mathrm{~nm},{ }^{23}$ and a volume charge density of $-1300 \mathrm{~mol} e \mathrm{~m}^{-3}$ at $\mathrm{pH} 8 .{ }^{47}{ }^{c}$ Computed using the NICA-Donnan generic parameters ${ }^{28}$ for the given total concentrations of $\mathrm{Ca}^{2+}$ and HA.

whilst $\mathrm{Pb}$ (II) and $\mathrm{Cu}$ (II) are approximately equally distributed between these two forms. Furthermore, the total concentration of $\mathrm{Cd}$ (II), $\mathrm{Pb}$ (II) and $\mathrm{Cu}$ (II) associated with the particles is similar at $I=100$ and $10 \mathrm{~mol} \mathrm{~m}^{-3}$. In contrast, in practically all cases considered the NICA-Donnan model predicts that the vast majority of the intraparticulate $\mathrm{M}$ is in the form of innersphere complexes (Table 3). It also predicts a much greater total concentration in the particle body at an ionic strength of $100 \mathrm{~mol} \mathrm{~m}^{-3}$ as compared to $10 \mathrm{~mol} \mathrm{~m}^{-3}$. This outcome is a consequence of the ionic strength dependence of the NICADonnan $V_{\mathrm{D}}$ value which translates to, for equivalent total mass in the dispersion, the volume fraction of HA being a factor of 6 times lower at an ionic strength of $100 \mathrm{~mol} \mathrm{~m}^{-3}$ as compared to $10 \mathrm{~mol} \mathrm{~m}^{-3}$, in contradiction with experimental findings (see Section 4.1).

The extent to which $\mathrm{Ca}^{2+}$ electrostatically associates with $\mathrm{HA}$ provides a useful means to probe the consistency of the speciation results for $\mathrm{Cd}^{2+}, \mathrm{Pb}^{2+}$ and $\mathrm{Cu}^{2+}$ given in Table 3 . That is, the deliberate use of a calcium salt, e.g. $\mathrm{Ca}\left(\mathrm{NO}_{3}\right)_{2}$, as the background electrolyte, with a concentration of $\mathrm{Ca}^{2+}$ several orders of magnitude greater than that of the target $\mathrm{M}^{2+}$, enables the electrostatic contribution to the binding of $\mathrm{M}^{2+}$ to be eliminated. This feature is a consequence of the purely electrostatic counterion condensation demands of the HA entity being practically totally met by the abundant $\mathrm{Ca}^{2+}$. Accordingly, in terms of the CCD approach the intraparticulate speciation of $\mathrm{M}^{2+}$ will involve only Donnan-type partitioning in $V_{\mathrm{D}}$, Boltzmann accumulation in $V_{\mathrm{DL}}$ and inner-sphere complexation in $V_{\mathrm{D}}$ and $V_{\mathrm{DL}}$. It should be noted that for a given ionic strength, the effective Donnan potential in the $2: 1$ electrolyte is lower than in the standard 1:1 electrolyte (cf. eqn (5)). For comparison, computations were performed with the NICA-Donnan model in $\mathrm{Ca}\left(\mathrm{NO}_{3}\right)_{2}$ media for the same bulk concentrations of $\mathrm{HA}$ and $\mathrm{M}$ (II) as used in the experiments reported herein.

Several factors must be taken into account in comparing the $\bar{K}_{\text {int }}$ values derived for the different ionic strengths in $\mathrm{KNO}_{3}$ vs. $\mathrm{Ca}\left(\mathrm{NO}_{3}\right)_{2}$ electrolyte. As described above, in $\mathrm{Ca}\left(\mathrm{NO}_{3}\right)_{2}$ there is no counterion condensation of the target $\mathrm{M}^{2+}$ and the Donnan potential is lower than that in $\mathrm{KNO}_{3}$. Accordingly, the intraparticulate environments in $\mathrm{KNO}_{3}$ vs. $\mathrm{Ca}\left(\mathrm{NO}_{3}\right)_{2}$ electrolyte differ in terms of the net charge density and the corresponding $\psi_{\mathrm{D}}$, the ensuing magnitude of $\bar{f}_{\mathrm{B}, \mathrm{M}}$ for the intraparticulate free $\mathrm{M}^{2+}$, and the effective charge screening. In comparing the behavior of $\mathrm{Cd}(\mathrm{II}), \mathrm{Pb}$ (II) and $\mathrm{Cu}(\mathrm{II})$, we must also take into account the different chemical affinities of these transition metal ions for the reactive sites as well as the heterogeneous nature of the HA complexant. The chemical affinity of the target $\mathrm{M}^{2+}$ towards the major complexing groups of HA follows the order $\mathrm{Cd}(\mathrm{II})<$ $\mathrm{Pb}(\mathrm{II}) \approx \mathrm{Cu}$ (II), whilst their degree of heterogeneity follows $\mathrm{Cd}(\mathrm{II})<\mathrm{Pb}(\mathrm{II})<\mathrm{Cu}(\mathrm{II}) .{ }^{1}$ The overall consequence for each $\mathrm{M}^{2+}$ is that $\bar{K}_{\text {int }}$ decreases as $\theta_{\mathbf{M}}$ increases, with the magnitude of this effect being governed by the heterogeneity of the complexes. That is, for a given increase in $\theta_{\mathbf{M}}$, the $\bar{K}_{\text {int }}$ for $\mathrm{Cu}(\mathrm{II})$ is expected to decrease by a greater extent than that for $\mathrm{Cd}(\mathrm{II})$.

With the above features in mind, we consider the physicochemical reasonableness of the CCD and NICA-Donnan results. The case of $\mathrm{Cd}(\mathrm{II})$ at $I=100 \mathrm{~mol} \mathrm{~m}^{-3}$ provides a strong test of the consistency of the two approaches: at the metal-to-site ratio considered, inner-sphere binding is rather weak, and both

Table 3 Comparison of intraparticulate speciation obtained via the CCD and NICA-Donnan (NICAD) models for $\mathrm{KNO}_{3}$ electrolyte. $c_{\mathrm{M}, \mathrm{t}}^{*}=4.6 \times 10^{-3} \mathrm{~mol} \mathrm{~m}^{-3} ; c_{\mathrm{HA}, \mathrm{t}}^{*}=50 \mathrm{~g} \mathrm{~m}^{-3} ; \mathrm{pH}=6$ in bulk electrolyte medium

\begin{tabular}{|c|c|c|c|c|c|c|c|c|c|c|c|c|}
\hline & \multicolumn{2}{|c|}{$\begin{array}{l}\mathrm{Cd}, \\
I=100 \mathrm{~mol} \mathrm{~m}^{-3}\end{array}$} & \multicolumn{2}{|c|}{$\begin{array}{l}\mathrm{Cd}, \\
I=10 \mathrm{~mol} \mathrm{~m}^{-3}\end{array}$} & \multicolumn{2}{|c|}{$\begin{array}{l}\mathrm{Pb}, \\
I=100 \mathrm{~mol} \mathrm{~m}^{-3}\end{array}$} & \multicolumn{2}{|c|}{$\begin{array}{l}\mathrm{Pb}, \\
I=10 \mathrm{~mol} \mathrm{~m}^{-3}\end{array}$} & \multicolumn{2}{|c|}{$\begin{array}{l}\mathrm{Cu}, \\
I=100 \mathrm{~mol} \mathrm{~m}^{-3}\end{array}$} & \multicolumn{2}{|c|}{$\begin{array}{l}\mathrm{Cu}, \\
I=10 \mathrm{~mol} \mathrm{~m}^{-3}\end{array}$} \\
\hline & CCD & NICAD & CCD & NICAD & CCD & NICAD & CCD & NICAD & CCD & NICAD & CCD & NICAD \\
\hline$c_{\mathrm{M}, \mathrm{f}}^{*}, \mathrm{~mol} \mathrm{~m}^{-3}$ & $1 \times 10^{-3}$ & $1.8 \times 10^{-3}$ & $3 \times 10^{-4}$ & $5.7 \times 10^{-4}$ & $3 \times 10^{-7}$ & $4.1 \times 10^{-5}$ & $6.5 \times 10^{-8}$ & $9.5 \times 10^{-6}$ & $7 \times 10^{-7}$ & $3.6 \times 10^{-6}$ & $4 \times 10^{-7}$ & $9.1 \times 10^{-7}$ \\
\hline$c_{\mathrm{M}, \mathrm{t}}, \mathrm{mol} \mathrm{m}^{-3}$ & 28.6 & 54.4 & 34.1 & 27.2 & 36.5 & 95.0 & 36.5 & 31.1 & 36.5 & 96.2 & 36.5 & 31.2 \\
\hline Fraction free in $V_{\mathrm{DL}}$ & $3 \times 10^{-5}$ & - & $2 \times 10^{-4}$ & - & $8 \times 10^{-9}$ & - & $3.5 \times 10^{-8}$ & - & $2 \times 10^{-8}$ & - & $2 \times 10^{-7}$ & - \\
\hline Fraction free in $V_{\mathrm{D}}$ & $2 \times 10^{-3}$ & 0.03 & 0.04 & 0.16 & $4 \times 10^{-7}$ & $4 \times 10^{-4}$ & $8 \times 10^{-6}$ & $2.4 \times 10^{-3}$ & $9 \times 10^{-7}$ & $3.5 \times 10^{-5}$ & $5 \times 10^{-5}$ & $2.3 \times 10^{-4}$ \\
\hline $\begin{array}{l}\text { Fraction } \\
\text { condensed in } V_{\mathrm{DL}}\end{array}$ & 0.7 & - & 0.55 & - & 0.5 & - & 0.5 & - & 0.5 & - & 0.5 & - \\
\hline $\begin{array}{l}\text { Fraction } \\
\text { inner-sphere MS }\end{array}$ & 0.3 & 0.97 & 0.41 & 0.84 & 0.5 & 1 & 0.5 & 0.998 & 0.5 & 1 & 0.5 & 1 \\
\hline$\theta_{\mathrm{M}}$ & 0.014 & 0.018 & 0.02 & 0.026 & 0.026 & 0.03 & 0.026 & 0.036 & 0.026 & 0.033 & 0.026 & 0.035 \\
\hline $\bar{f}_{\mathrm{B}, \mathrm{M}}$ in $V_{\mathrm{D}}$ & 50 & 950 & 5000 & 7700 & 50 & 930 & 5000 & 7700 & 50 & 940 & 5000 & 7800 \\
\hline $\begin{array}{l}\log \bar{K}_{\mathrm{int}} \\
\left(\mathrm{m}^{3} \mathrm{~mol}^{-1}\right)^{a}\end{array}$ & -0.52 & -2.0 & -1.8 & -2.2 & 3.2 & -0.1 & 2 & -0.3 & 3 & 1.0 & 1.3 & 0.7 \\
\hline
\end{tabular}


models show that the total concentration of intraparticulate $\mathrm{Cd}(\mathrm{II})$ is significantly reduced in the presence of excess $\mathrm{Ca}^{2+}$ (Tables 3 and 4). The CCD approach generates similar values for both $\theta_{\mathrm{Cd}}$ and the intraparticulate concentration of free $\mathrm{Cd}^{2+}$ $\left(=\bar{f}_{\mathrm{B}, \mathrm{M}} c_{\mathrm{M}, \mathrm{f}}^{*}\right)$ in the two electrolytes, and consequently similar magnitudes of $\bar{K}_{\text {int }}$. The total concentration of intraparticulate $\mathrm{Cd}$ (II) in $\mathrm{Ca}\left(\mathrm{NO}_{3}\right)_{2}$ is lower than in $\mathrm{KNO}_{3}$ by a factor of $c a .1 / 3$, which reflects the absence of the condensed fraction (comprising $70 \%$ of the total intraparticulate $\mathrm{Cd}(\mathrm{II})$ in $\mathrm{KNO}_{3}$; Table 3). Furthermore, we note that the $\bar{K}_{\text {int }}$ value is comparable to the intrinsic affinity of simple carboxylate ligands, which is physicochemically reasonable for $\mathrm{Cd}(\mathrm{II})$ under these conditions, e.g. $K_{\text {int }}$ (eqn (4)) for Cd-acetate is of the order $0.01 \mathrm{~m}^{3} \mathrm{~mol}^{-1}{ }^{-48,49}$ The NICA-Donnan derived $\bar{K}_{\text {int }}$ values for $\mathrm{Cd}$ (II) are in broad agreement with the CCD result, being also of order $0.01 \mathrm{~mol} \mathrm{~m}^{-3}$ in both electrolytes. However, detailed examination reveals discrepancies in terms of the physicochemical reasonableness of the underlying parameters. Firstly, the concentration of intraparticulate free $\mathrm{M}^{2+}$ is a factor of $c a .10$ higher in $\mathrm{KNO}_{3}$ than in $\mathrm{Ca}\left(\mathrm{NO}_{3}\right)_{2}$. As a consequence, the $\theta_{\mathrm{Cd}}$ in $\mathrm{Ca}\left(\mathrm{NO}_{3}\right)_{2}$ electrolyte is much lower, and the derived $\log \bar{K}_{\text {int }}$ value is $c a$. half a unit higher. In addition, the decrease in the total concentration of intraparticulate $\mathrm{Cd}(\mathrm{II})$ in $\mathrm{Ca}\left(\mathrm{NO}_{3}\right)_{2}$ is much greater than can be explained by electrostatic factors alone, i.e. the reduction in magnitude of the Donnan potential. These outcomes are a consequence of the NICADonnan assumption that $\mathrm{Ca}^{2+}$ association involves significant innersphere complexation, with consequent displacement of $\mathrm{Cd}^{2+}$ from its inner-sphere complexes with weaker sites.

For $\mathrm{Cu}(\mathrm{II})$, both the magnitude and the heterogeneity of the intrinsic chemical affinity of its complexes with HA are greater than those for $\mathrm{Cd}(\mathrm{II})$. Again, some notable differences in consistency of the interpretation by the CCD and NICA-Donnan models are apparent. At $I=10 \mathrm{~mol} \mathrm{~m} \mathrm{~m}^{-3}$, the CCD approach shows that both $\theta_{\mathrm{Cu}}$ and the intraparticulate concentration of free $\mathrm{Cu}^{2+}$ are significantly higher in $\mathrm{Ca}\left(\mathrm{NO}_{3}\right)_{2}$ electrolyte than in $\mathrm{KNO}_{3}$. The total concentration of intraparticulate $\mathrm{Cu}(\mathrm{II})$ is approximately the same in both electrolytes, whilst the speciation changes from approximately equal amounts of condensed ions and inner-sphere complexes in $\mathrm{KNO}_{3}$ (Table 3) to practically only inner-sphere complexes in $\mathrm{Ca}\left(\mathrm{NO}_{3}\right)_{2}$ (Table 4). The $\bar{K}_{\text {int }}$ is $c a$. an order of magnitude lower in $\mathrm{Ca}\left(\mathrm{NO}_{3}\right)_{2}$ than in $\mathrm{KNO}_{3}$ which predominantly reflects the $c a$. one order of magnitude higher $\theta_{\mathrm{Cu}}$. This result reflects the heterogeneity of the HA complexant: a greater proportion of intraparticulate innersphere complexes concomitantly means that the average affinity of the complexes is lower. NICA-Donnan derived results for $\mathrm{Cu}$ (II) at $I=10 \mathrm{~mol} \mathrm{~m}^{-3}$ show that the total concentration of intraparticulate $\mathrm{Cu}(\mathrm{II})$ and $\theta_{\mathrm{Cu}}$ are the same in both electrolytes, and almost all of the particle-associated $\mathrm{Cu}$ (II) is predicted to be in the form of inner-sphere complexes in both cases. However, the intraparticulate concentration of free $\mathrm{Cu}^{2+}$ is a factor of $c a .3$ lower in $\mathrm{Ca}\left(\mathrm{NO}_{3}\right)_{2}$. The lower concentration of free $\mathrm{Cu}^{2+}$ within the particle body has the consequence that the $\bar{K}_{\text {int }}$ values obtained in $\mathrm{Ca}\left(\mathrm{NO}_{3}\right)_{2}$ are greater than those in $\mathrm{KNO}_{3}$ (by $c a$. half a $\log$ unit). Others have reported that the presence of $\mathrm{Ca}^{2+}$ reduces




the extent to which $\mathrm{Cd}(\mathrm{II})$ is bound by HA, but has negligible impact on the extent of $\mathrm{Cu}$ (II)-HA complex formation. ${ }^{50,51}$ In the context of the NICA-Donnan model the inconsistent observation was explained by saying that $\mathrm{Ca}^{2+}$ hardly impacts on $\mathrm{Cu}-\mathrm{HA}$ binding because the inner-sphere complexes of $\mathrm{Cu}$ (II) with phenolic type sites are stronger than those of $\mathrm{Ca}(\mathrm{II})$, whilst in the case of $\mathrm{Cd}(\mathrm{II}), \mathrm{Ca}^{2+}$ can compete for inner-sphere complexation of carboxyl groups. ${ }^{50}$

\section{Conclusions and outlook}

Over the years a number of models have been developed to describe metal ion binding by HA. Each approach has provided insights into the nature of the association of ions with this charged, chemically heterogeneous complexant. Recent advances in understanding the physicochemical properties of soft, charged nanoparticles cast new light on M-HA complexation phenomena. In accounting for the electrostatic features of soft charged NPs such as HA, a purely Donnan type approach is demonstrated to be a physically incomplete description. Notably, Donnan partitioning by itself greatly underestimates the extent to which $\mathrm{Ca}^{2+}$ associates with HA, and even though covalency is essentially absent in $\mathrm{Ca}^{2+}$ binding, conventional equilibrium speciation models resort to inclusion of chemical affinity parameters to describe the $\mathrm{Ca}^{2+}-\mathrm{HA}$ association. Taking the NICA-Donnan approach as an example, there are several factors in the model that are out of line with experimental observations. ${ }^{34,35}$ These include e.g. the values of the protonation constants, the delineation and the ionic strength dependence of the Donnan volume, as well the magnitude of the Donnan potential. Each of the disparities acts to increase the extent of association of oppositely charged ions with the HA entity, thus pointing to possibly missing electrostatic ingredients in the interpretation. It is evident that the $\mathrm{Ca}^{2+}-\mathrm{HA}$ association involves stronger electrostatic interactions such as counterion condensation, potentially including the possibility for partial formation of inner-sphere ion pairs in which $\mathrm{Ca}_{\mathrm{aq}}{ }^{2+}$ exchanges some of its inner-sphere water of hydration. ${ }^{52,53}$ The CCD approach is shown to provide a more satisfactory physicochemical description of the electrostatic contribution to association of $\mathrm{M}^{2+}$ with HA.

There are significant differences in the intraparticulate speciation predicted by the CCD and NICA-Donnan approaches. Notably, in 1-1 electrolyte the CCD approach finds a substantial electrostatic contribution to the association of $\mathrm{Cd}(\mathrm{II}), \mathrm{Pb}$ (II), and $\mathrm{Cu}$ (II) with HA. It follows that at the level of the $\bar{K}_{\text {int }}$ values, the CCD and Donnan-only electrostatic models give significantly different results. For the heterogeneous HA complexant, $\bar{K}_{\text {int }}$ is expected to increase as $\theta_{\mathbf{M}}$ decreases. The CCD model retrieves this relationship, whilst the trend in the NICA-Donnan derived values is less systematic. Such differences in intraparticulate speciation are highly significant for e.g. predictions of the lability and bioavailability of the complex species. For example, the present work has shown that for $\mathrm{Pb}-\mathrm{HA}$ the total concentration of intraparticulate $\mathrm{Pb}$ (II) is similar in $\mathrm{KNO}_{3}$ and $\mathrm{Ca}\left(\mathrm{NO}_{3}\right)_{2}$ electrolyte, yet the lability of the complexes is very different in these two media, as a consequence of effect of the electrostatic environment in the NP body on the reaction rate constants. ${ }^{54}$
A detailed exploration of the consequences of condensation phenomena on the intraparticulate dynamics of M-HA complexes is underway.

\section{Symbols and abbreviations}

$\begin{array}{ll}\text { CC } & \text { Counterion condensation } \\ \text { D } & \text { Donnan phase } \\ \text { DL } & \text { Double layer } \\ \text { HA } & \text { Humic acid } \\ I & \text { Ionic strength } \\ \text { S } & \text { Reactive site } \\ \text { NP } & \text { Nanoparticle }\end{array}$

SSCP Stripping chronopotentiometry at scanned deposition potential

$c_{\mathrm{M}, \mathrm{f}}^{*} \quad$ Concentration of free metal ion in bulk electrolyte medium $\left(\mathrm{mol} \mathrm{m}^{-3}\right)$

$c_{\mathrm{M}, \mathrm{t}}^{*} \quad$ Total concentration of metal in the entire dispersion $\left(\mathrm{mol} \mathrm{m}^{-3}\right)$

$c_{\mathrm{M}, \mathrm{f}}^{\mathrm{DL}} \quad$ Local concentration of free metal ion in the intraparticulate double layer $\left(\mathrm{mol} \mathrm{m}^{-3}\right)$

$c_{\mathrm{M}, \mathrm{f}}^{\mathrm{D}} \quad$ Local concentration of free metal ion in the Donnan volume $\left(\mathrm{mol} \mathrm{m}^{-3}\right)$

$c_{\mathrm{M} \text {,cond }}^{\mathrm{DL}} \quad$ Local concentration of condensed metal ion in the intraparticulate double layer $\left(\mathrm{mol} \mathrm{m}^{-3}\right)$

$c_{\mathrm{HA}, \mathrm{t}}^{*} \quad$ Total smeared-out concentration of HA in the aqueous dispersion $\left(\mathrm{g} \mathrm{m}^{-3}\right)$

$c_{\mathrm{M}, \mathrm{f}} \quad$ Local average concentration of free metal ion in the particle body $\left(\varphi_{\mathrm{DL}} c_{\mathrm{M}, \mathrm{f}}^{\mathrm{DL}}+\varphi_{\mathrm{D}} c_{\mathrm{M}, \mathrm{f}}^{\mathrm{D}}\right)\left(\mathrm{mol} \mathrm{m}^{-3}\right)$

$c_{\mathrm{M}, \mathrm{t}} \quad$ Local total concentration of all forms of $\mathrm{M}$ in the particle body $\left(\mathrm{mol} \mathrm{m}^{-3}\right)$

$c_{\text {MS }} \quad$ Local concentration of inner-sphere complex within the particle volume $\left(\mathrm{mol} \mathrm{m}^{-3}\right)$

$c_{\mathrm{MS}}^{*} \quad$ Smeared-out concentration of inner-sphere bound $\mathbf{M}$ $\left(\mathrm{mol} \mathrm{m}^{-3}\right)$

$c_{\mathrm{S}, \mathrm{t}}^{*} \quad$ Smeared-out total concentration of reactive sites $\left(\mathrm{mol} \mathrm{m}^{-3}\right)$

$c_{\mathrm{S}}^{*} \quad$ Smeared-out concentration of reactive sites $\left(\mathrm{mol} \mathrm{m}^{-3}\right)$

$c_{\mathrm{S}, \mathrm{t}} \quad$ Local total concentration of reactive sites within the particle volume $\left(\mathrm{mol} \mathrm{m}^{-3}\right)$

$c_{\mathrm{S}} \quad$ Local concentration of reactive sites within the particle volume $\left(\mathrm{mol} \mathrm{m}^{-3}\right)$

Elementary charge

$\bar{f}_{\mathrm{B}, \mathrm{M}}$

$\bar{f}_{\mathrm{C}}$

Donnan partitioning factor for the metal ion

Condensation factor in the intraparticulate double layer Concentration enhancement factor for $\mathrm{M}^{z+}$ in the outer-sphere volume of an individual ion-pair in simple ligand case

$K \quad$ Conventional stability constant for a simple ligand $\left(\mathrm{m}^{3} \mathrm{~mol}^{-1}\right)$

$K^{\mathrm{os}} \quad$ Outer-sphere ion pair stability constant $\left(\mathrm{m}^{3} \mathrm{~mol}^{-1}\right)$

$\bar{K}_{\text {app }} \quad$ Apparent average stability constant for MHA based on smeared-out concentrations $\left(\mathrm{m}^{3} \mathrm{~mol}^{-1}\right)$

$\bar{K}_{\text {int }} \quad$ Intrinsic average stability constant for inner-sphere MS $\left(\mathrm{m}^{3} \mathrm{~mol}^{-1}\right)$ 
$\kappa^{-1} \quad$ Debye length in the bulk aqueous medium (m)

$\kappa_{\mathrm{p}}^{-1} \quad$ Intraparticulate Debye length (m)

$\ell_{\mathrm{DL}} \quad$ Thickness of the intraparticulate condensation zone (m)

$\ell_{\mathrm{C}} \quad$ Separation distance between charged sites $(\mathrm{m})$

$n_{\mathrm{S}} \quad$ Number of reactive sites per particle

$r_{\mathrm{p}} \quad$ Particle radius $(\mathrm{m})$

$V_{\mathrm{D}} \quad$ Volume of the Donnan phase $\left(\mathrm{m}^{3}\right)$

$V_{\text {DL }} \quad$ Volume of the intraparticulate double layer zone $\left(\mathrm{m}^{3}\right)$

$V^{\text {os }} \quad$ Outer-sphere volume for an ion pair between $\mathrm{M}_{\mathrm{aq}}{ }^{2+}$ and an individual site $\mathrm{S}\left(\mathrm{m}^{3}\right)$

$V_{\mathrm{p}} \quad$ Volume of the NP entity $\left(\mathrm{m}^{3}\right)$

$\psi_{\mathrm{D}} \quad$ Donnan potential (V)

$\psi_{\mathrm{DL}} \quad$ Potential at a given spatial position within the intraparticulate double layer $(\mathrm{V})$

$\bar{\rho}_{\mathrm{DL}} \quad$ Average volume charge density in the double layer $\left(\mathrm{mol} e \mathrm{~m}^{-3}\right.$ )

$\rho_{\mathrm{p}} \quad$ Structural volume charge density in the particle body $\left(\mathrm{mol} e \mathrm{~m}^{-3}\right.$ )

$\varphi_{\text {DL }} \quad$ Particle volume fraction of the double layer zone

$\varphi_{\mathrm{D}} \quad$ Particle volume fraction of the Donnan zone

$\theta_{\mathbf{M}} \quad$ Ratio of concentrations of inner-sphere complexes and reactive sites

\section{References}

1 J. Buffle, Complexation Reactions in Aquatic Systems: An Analytical Approach, Ellis Horwood, Chichester, UK, 1988.

2 L. K. Koopal, T. Saito, J. P. Pinheiro and W. H. van Riemsdijk, Colloids Surf., A, 2005, 265, 40-54.

3 E. Tipping, Aquat. Geochem., 1998, 4, 3-48.

4 J. F. L. Duval, K. J. Wilkinson, H. P. van Leeuwen and J. Buffle, Environ. Sci. Technol., 2005, 39, 6435-6445.

5 H. P. van Leeuwen and J. Buffle, Environ. Sci. Technol., 2009, 43, 7175-7183.

6 R. M. Town, J. F. L. Duval, J. Buffle and H. P. van Leeuwen, J. Phys. Chem. A, 2012, 116, 6489-6496.

7 P. Warwick, A. Hall, S. J. King, J. Zhu and J. van der Lee, Radiochim. Acta, 1998, 81, 215-221.

8 A. P. Robertson and J. O. Leckie, Environ. Sci. Technol., 1999, 33, 786-795.

9 R. Marsac, M. Davranche, G. Gruau, M. Bouhnik-Le Coz and A. Dia, Geochim. Cosmochim. Acta, 2011, 75, 5625-5637.

10 A. C. Montenegro, S. Orsetti and F. V. Molina, Environ. Chem., 2014, 11, 318-332.

11 N. D. Bryan, D. M. Jones, M. Appleton, F. R. Livens, M. N. Jones, P. Warwick, S. King and A. Hall, Phys. Chem. Chem. Phys., 2000, 2, 1291-1300.

12 M. F. Benedetti, W. H. van Riemsdijk and L. K. Koopal, Environ. Sci. Technol., 1996, 30, 1805-1813.

13 I. Christl, A. Metzger, I. Heidmann and R. Kretzschmar, Environ. Sci. Technol., 2005, 39, 5319-5326.

14 A. Liu and R. D. Gonzalez, Langmuir, 2000, 16, 3902-3909.

15 H. P. van Leeuwen and R. M. Town, Environ. Chem., 2016, 13, 76-83.
16 G. S. Manning, Biophys. Chem., 1977, 7, 95-102.

17 G. S. Manning, Q. Rev. Biophys., 1978, 2, 179-246.

18 E. Garcia-Fernandez, P. M. R. Paulo and S. M. B. Costa, Phys. Chem. Chem. Phys., 2015, 17, 4319-4327.

19 M. Majtyka and J. Kłos, Phys. Chem. Chem. Phys., 2007, 9, 2284-2292.

20 Q. R. Huang, P. L. Dubin, C. N. Moorefield and G. R. Newkome, J. Phys. Chem. B, 2000, 104, 898-904.

21 Z. Li, A. K. van Dyk, S. J. Fitzwater, K. A Fichthorn and S. T. Milner, Langmuir, 2016, 32, 428-441.

22 H. P. van Leeuwen, R. F. M. J. Cleven and P. Valenta, Pure Appl. Chem., 1991, 63, 1251-1268.

23 R. M. Town and H. P. van Leeuwen, Phys. Chem. Chem. Phys., 2016, 18, 10049-10058.

24 R. M. Fuoss, J. Am. Chem. Soc., 1958, 80, 5059-5061.

25 H. P. van Leeuwen, R. M. Town and J. Buffle, J. Phys. Chem. A, 2007, 111, 2115-2121.

26 H. Ohshima and T. Kondo, Biophys. Chem., 1990, 38, 117-122.

27 L. P. Yezek and H. P. van Leeuwen, Langmuir, 2005, 21, 10342-10347.

28 C. J. Milne, D. G. Kinniburgh, W. H. van Riemsdijk and E. Tipping, Environ. Sci. Technol., 2003, 37, 958-971.

29 M. J. Avena, L. K. Koopal and W. H. van Riemsdijk, J. Colloid Interface Sci., 1999, 217, 37-48.

30 W. F. Tan, L. K. Koopal and W. Norde, Environ. Sci. Technol., 2009, 43, 591-596.

31 J. P. Pinheiro, A. M. Mota, J. M. R. d'Oliveira and J. M. G. Martinho, Anal. Chim. Acta, 1996, 329, 15-24.

32 M. Hosse and K. J. Wilkinson, Environ. Sci. Technol., 2001, 35, 4301-4306.

33 J. F. L. Duval, Langmuir, 2005, 21, 3247-3258.

34 T. Lenoir, A. Matynia and A. Manceau, Environ. Sci. Technol., 2010, 44, 6221-6227.

35 E. Companys, J. L. Garcés, J. Salvador, J. Galceran, J. Puy and F. Mas, Colloids Surf., A, 2007, 306, 2-13.

36 D. G. Kinniburgh, W. H. van Riemsdijk, L. K. Koopal, M. Borkovec, M. F. Benedetti and M. J. Avena, Colloids Surf., A, 1999, 151, 147-166.

37 J. F. L. Duval and H. P. van Leeuwen, J. Phys. Chem. A, 2012, 116, 6443-6451.

38 I. Christl, A. Metzger, I. Heidmann and R. Kretzschmar, Environ. Sci. Technol., 2005, 39, 5319-5326.

39 L. Weng, E. J. M. Temminghoff, S. Lofts, E. Tipping and W. H. van Riemsdijk, Environ. Sci. Technol., 2002, 36, 4804-4810.

40 K. Krishnan and R. A. Plane, J. Am. Chem. Soc., 1968, 90, 3195-3200.

41 L. A. Clapp, C. J. Siddons, J. R. Whitehead, D. G. van Derveer, R. D. Rogers, S. T. Griffin, S. B. Jones and R. D. Hancock, Inorg. Chem., 2005, 44, 8495-8502.

42 C. Travers and J. A. Marinsky, J. Polym. Sci., 1974, 47, 285-297.

43 I. Christl, Environ. Chem., 2012, 9, 89-96.

44 L. A. Oste, E. J. M. Temminghoff, T. M. Lexmond and W. H. van Riemsdijk, Anal. Chem., 2002, 74, 856-862. 
45 J. Buffle, Z. Zhang and K. Startchev, Environ. Sci. Technol., 2007, 41, 7609-7620.

46 J. G. Hering and F. M. M. Morel, Environ. Sci. Technol., 1988, 22, 1234-1237.

47 W.-F. Tan, W. Norde and L. K. Koopal, Geochim. Cosmochim. Acta, 2011, 75, 5749-5761.

48 A. Yuchi, H. Wada and G. Nakagawa, Anal. Chim. Acta, 1983, 149, 209-216.

49 R. S. Kolat and J. E. Powell, Inorg. Chem., 1962, 1, 293-296.
50 D. G. Kinniburgh, C. J. Milne, M. F. Benedetti, J. P. Pinheiro, J. Filius, L. K. Koopal and W. H. van Riemsdijk, Environ. Sci. Technol., 1996, 30, 1687-1698.

51 J. Hamilton-Taylor, A. S. Postill, E. Tipping and M. P. Harper, Geochim. Cosmochim. Acta, 2002, 66, 403-415.

52 F. J. Millero, F. Gombar and J. Oster, J. Solution Chem., 1977, 6, 269-280.

53 L. M. Hamm, A. F. Wallace and P. M. Dove, J. Phys. Chem. B, 2010, 114, 10488-10495.

54 R. M. Town, Environ. Chem., 2015, 12, 130-137. 